\title{
Association of local anesthesia versus conscious sedation with functional outcome of acute ischemic stroke patients undergoing embolectomy
}

\section{Joseph Marion, MD 1; Seyed Seyedsaadat, MD 2; Jeffery Pasternak, MD 3;}

Alejandro Rabinstein, MD 4; David Kallmes, MD 1; Waleed Brinjikji, MD 1

\section{Purpose}

Compare functional outcomes of acute ischemic stroke (AIS) patients undergoing embolectomy with either local anesthesia or conscious sedation. Secondarily, identify differences in hemodynamic parameters and complication rates between groups.

\section{Materials and Methods}

Single institution, retrospective review of all AIS patients undergoing embolectomy between January 2014 and July 2018 $(n=185)$. Patients receiving general anesthesia $(n=27)$ were excluded. 111 of 158 (70.3\%) composed the local anesthesia (LA) group, and 47 (29.7\%) composed the conscious sedation (CS) group. Median age was 71 years (IQR 59-79). 78 (49.4\%) were male. The median NIHSS score was 17.5 (IQR 11-21). Hemodynamic, medication, complication and functional outcome data were collected from the anesthesia and medical records. Good functional outcome was defined as a 3 month $\mathrm{mRS}<2$. A multivariate analysis was performed to estimate the association of anesthesia type on 3 month mRS score.

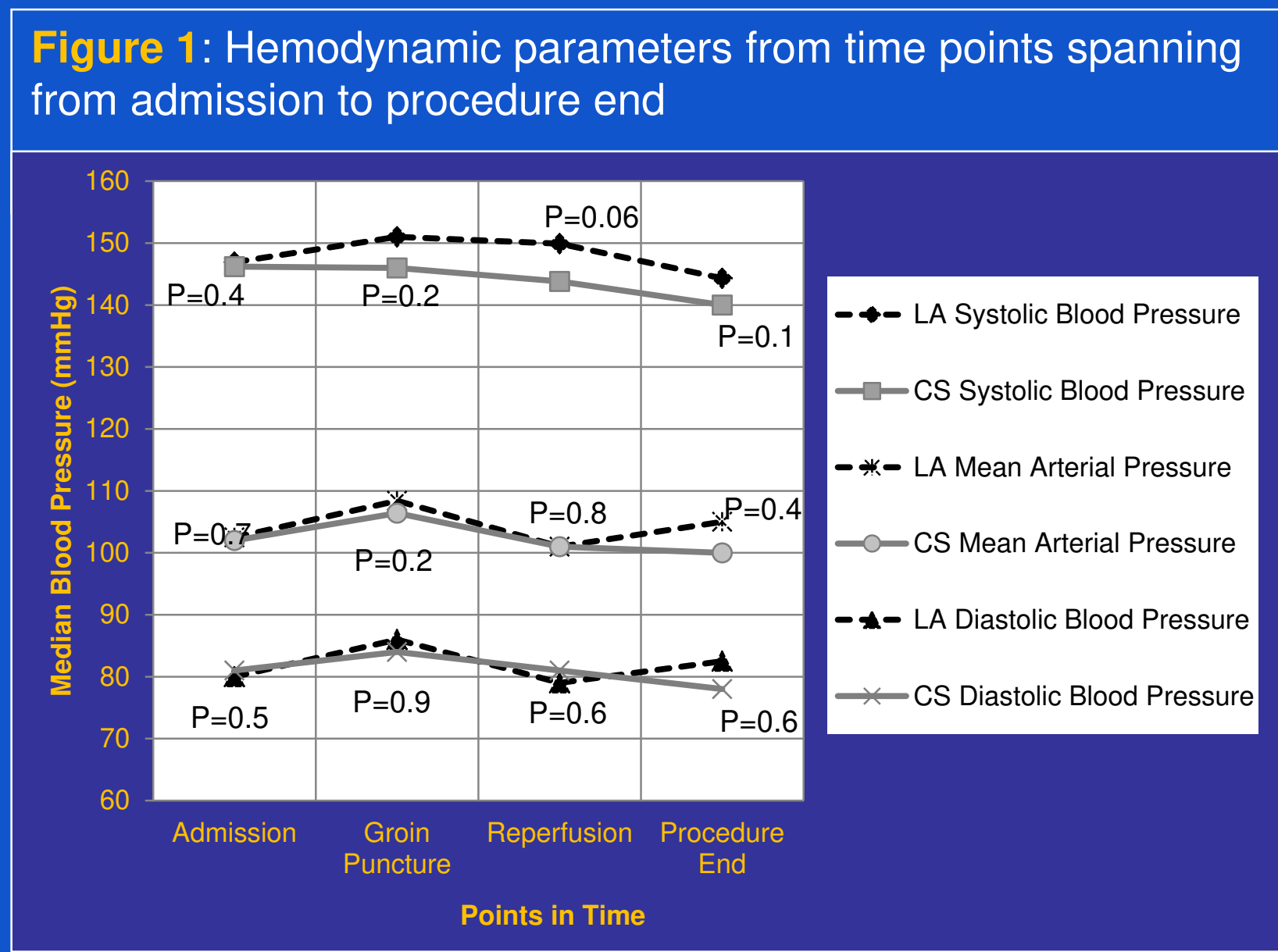

Results

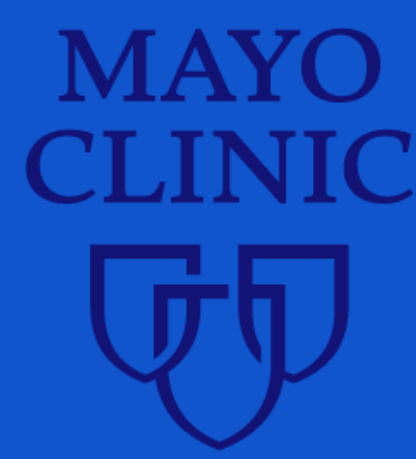

Three month mRS score $<2$ was similar between groups in both univariate and multivariate analyses $(p=0.5)$ (Table 1 and 2$)$. Younger patients were more likely to receive conscious sedation $(p=0.01)$. CS patients were more likely to receive intravenous thrombolytic prior to embolectomy $(p=0.025)$. The complication rate and hemodynamic parameters were similar between groups (Figure 1).

\begin{tabular}{|l|c|c|c|c|c|c|}
\hline \multicolumn{6}{|c|}{ Table 1: Univariate analysis of the effect of local anesthesia (LA) } \\
vs. conscious sedation (CS) on the outcome of AIS patients. \\
\hline \hline
\end{tabular}

\begin{tabular}{|l|c|c|c|}
\hline \multicolumn{4}{|c|}{ Table 2: Multivariate analysis predicting functional outcome } \\
\hline & $\begin{array}{l}\text { Effect } \\
\text { Measure }\end{array}$ & Effect value (95\% CI) & P - Value \\
\hline Age & OR & $0.96(0.93-0.99)$ & 0.02 \\
\hline Post treatment ICH & OR & $6.83(1.96-23.74)$ & 0.003 \\
\hline Pretreatment NIHSS score & OR & $0.93(0.87-0.99)$ & 0.04 \\
\hline Sedation type (LA versus CS) & OR & $1.3(0.47-3.49)$ & 0.6 \\
\hline
\end{tabular}

\section{Conclusion}

Functional outcome of AIS patients undergoing embolectomy is similar for patients receiving local anesthesia and conscious sedation. This similarity may be beneficial to a future study comparing general anesthesia to LA and CS. The use of LA or CS does not significantly impact hemodynamic status. 